\title{
SOBRE LA DIFUSIÓ DE LES OBRES DE PLEYEL A CATALUNYA
}

\author{
Josep M. VILAR
}

A Maria Ester-Sala, en el record

Per mitjà de les nostres recerques en un bon nombre d'arxius musicals de Catalunya' de titularitat i configuracions diverses, i repartits arreu de la geografia del país, hem pogut observar l'existència d'un volum molt considerable de còpies d'obres d'Ignaz Pleyel.

Aquest, juntament amb Franz Joseph Haydn i Gioacchino Rossini, és el compositor estranger que apareix més freqüentment en els arxius catalans consultats ${ }^{2}$.

Els casos de Haydn i de Pleyel són especialment significatius i interessants ja que corroboren l'existència d'una certa influència germànica en el classicisme català. Aquesta, inicialment fou negada o qüestionada en uns moments en els quals es tendí a considerar una omnipresència de la influència italiana. Posteriorment, diverses investigacions, entre les quals es compta aquesta mateixa, han posat l'èmfasi en la importància de la influència germànica que inicialment havia estat qüestionada i que posteriorment diferents investigacions han posat de manifest, fins al punt que en alguns casos s' ha pogut arribar a una certa sobrevaloració. Pensem que la presència, en terres de Catalunya, d'obres d'aquests dos grans mestres austríacs, però també d'altres figures menors tant d'aquell país com d'Alemanya testimonien que no sols van ser interpretades aquí sinó que van erigir-se en models de composició per als mestres de capella i organistes del país.

Aquesta seria, doncs, raó suficient per abordar una primera aproximació al coneixement d'aquest corpus de música tot esperant que la lenta catalogació dels fons musicals catalans que des de diferents fronts s'ha iniciat aporti un coneixement exhastiu d'aquestes obres, còpies i versions.

1. Aquest treball de recerca en més de 180 arxius d'arreu de Catalunya es va portar a terme, bàsicament, entre els anys 1984 i 1991 conjuntament amb Maria Ester-Sala. Alguns aspectes d'aquesta investigació es poden consultar a:

Ester, M.; Vilar, J. M., «Arxius Musicals a Catalunya». Revista Musical Catalana. Núm. 48 i ss. Barcelona, 1978 i ss.

-, -, «Una aproximació als fons de manuscrits musicals de Catalunya». Anuario Musical. Núm. 42 (1987), p. 229243; Núm. 44 (1989), p. 155-166; Núm. 46 (1991), p. 259-320.

-, -, «Els fons musicals a Catalunya: un patrimoni a revalorar». Lligall, 5 (1992), p. 123-146.

2. Vid.: Ester, M.; VILAR, J. M., «La presencia de Rossini en la vida cotidiana en la Catalunya del ochocientos». Nassarre, VIII, 2. (1992). p. 69-82. 
Però una segona raó ens ha mogut a escriure aquestes ratlles. És el fet que en el catàleg més complet que es té fins al moment de les obres de Pleyel $^{3}$ - molt complet però, si més no pel que fa a Catalunya, molt lluny de ser exhastiu-només hi ha consignat un sol arxiu de Catalunya que apareix a la llista inicial de sigles per utilitzar, però no als índexs finals. Es tracta de la Biblioteca de Catalunya.

Aquesta situació ve motivada, sens dubte, per la manca de catàlegs i inventaris que afecta a la majoria dels arxius catalans en l'actualitat.

En aquest article hem intentat fer una primera aproximació a la difusió que les obres de Pleyel van tenir a Catalunya. Així, s'han tingut en compte alguns aspectes que considerem especialment interessants mentre que s'han pasat per alt alguns altres que, sens dubte, hauran de ser objecte d'estudis ulteriors i més aprofundits. Entre aquests darrers hi ha les dates de les còpies, els copistes, els propietaris, les sèries o col-lecions $i$, sobretot, la confrontació de les obres anònimes amb el catàleg Pleyel. D'altra banda, també cal dir que s'ha identificat un cert nombre d'obres espúries o que - si més no- reben aquesta consideració des del moment que no apareixen al catàleg pres com a base del treball.

Per a la identificació de les obres ens hem basat en l'esmentat catàleg de Rita Benton. A aquesta obra fem referència en allò que concerneix a títols, numeració, etc. Les dades que fem constar la nostra llista són les següents:

Numeració de l'obra d'acord amb el catàleg de Rita Benton.

Pàgina d'aquest catàleg on es troba l'obra en qüestió.

Títol d'acord amb el catàleg. adjuntem).

Sigla de l'arxiu o biblioteca on es conserva la còpia (d'acord amb la taula de sigles que

Signatura del manuscrit (o altres numeracions) en el cas que en tingui.

Títol que figura a la còpia.

Instrumentació que figura a la còpia i que pot coincidir o no, amb l'original. S'han utilitzat diverses posibilitats genèriques:

Orquestra, sense especificació de la instrumentació.

Quintet (de corda).

Quartet (de corda).

Trio, que en tots els casos és de guitarra, violí i viola.

Duet, ja sigui de flautes o de violins.

Tecla. Només en els casos en què hi consta a la còpia es concreta si és per a piano o orgue. El tipus de còpia (manuscrit o imprès).

Altres comentaris com puguin ser els moviments concrets que figuren a la còpia quan l'obra no hi és sencera, l'edició exacta que s'ha trobat o bé la font impresa que probablement

3. Benton, Rita: Ignace Pleyel. A Thematic Catalogue of his Compositions. New York: Pendragon Press, 1977. 
va servir per a extreure'n les còpies manuscrites (indicat entre parèntesi i utilitzant la numeració de fonts - no d'obres-de Rita Benton), etc.

Les sigles d'arxius que s'han emprat són les següents:

Bac: Barcelona Arxiu de la Corona d'Aragó.

Bc: Barcelona. Biblioteca de Catalunya.

Bp: Barcelona, Esglesia de Santa Maria del Pi.

CE: Cervera. Arxiu Històric Comarcal.

ESm: Esparraguera. Arxiu Monné.

G: Girona. Arxiu de la Catedral.

MAs: Manresa. Arxiu de Música de la Seu.

MO: Montserrat. Arxiu del Monestir (manuscrits).

OLp: Olot. Parròquia de Sant Esteve.

RI: Ripoll. Museu-Arxiu.

SJ: Sant Joan de les Abadesses. Arxiu del Monestir.

SUe: Seu d'Urgell. Arxiu Episcopal.

TAG: Tàrrega. Arxiu Històric Comarcal.

VI: Vic. Arxiu Episcopal. (La numeració correspon a les pàgines de l'únic manuscrit musical existent d'aquest període).

VIG: Vilanova i la Geltrú. Parròquia de Santa Maria de La Geltrú. (La numeració correspon a les caixes arxivadores).

VPasm: Vilafranca del Penedès. Museu de Vilafranca. Arxiu de Preveres. (La numeració correspon al fons de música instrumental).

Altres abreviacions que s'hi han emprat són les següents:

prov.: provisional.

c.: caixa.

fl: flauta.

vl: violí.

vla: viola.

g: guitarra.

Del llistat que presentem es desprèn que, com a mínim, 138 composicions de Pleyel (sobre un total catalogat de 827 , és a dir una sisena part) van ser conegudes a Catalunya en el passat. Així i tot, la seva distribució per gèneres és molt irregular: mentre que aproximadament un $70 \%$ de les seves simfonies i quartets es van difondre per bona part del territori català, el nombre de duets i trios instrumentals que van arribar al país va ser molt menor. Per la seva banda, no hi ha cap constància que l'obra vocal i les composicions originals per a teclat hi tinguessin cap mena de difusió.

Ens inclinem a creure que sols un nombre relativamente limitat de les composicions de 
Pleyel devien tenir una difusió realmente significativa, i que les obres que trobem a Catalunya coincideixen a grans trets amb aquestes.

Creiem que és interessant $i$ aclaridor establir una correlació entre les composicions que es troben als diferents arxius catalans i les que apareixen consignades al Catálogo de impresos musicales del siglo XVIII en la Biblioteca Nacional ${ }^{4}$. A mes, segurament, que ambdós conjunts apleguen partitures que responen al gust i a la pràctica musical de dues comunitats més o menys concretes dins de l'Estat borbònic espanyol del segle XVIII. El resultat principal de la comparación és que hi ha gairebé un centenar d'obres que apareixen en ambdós llocs, la qual cosa representa aproximadament les tres quartes parts de cada un dels conjunts.

En aquests moments comença ja a ser coneguda la producció simfònica d'un reduït nombre de compositors catalans del període del classicismes, per la qual cosa, i d'acord amb el plantejament expresat abans, no sembla pas estrany que una societat que generà compositors interessats en la simfonia mostrés interès, també, per la importació d'obres d'aquest gènere. En canvi, i fins al moment, els nostres arxius segueixen sense oferir-nos mostres significatives de producció cambrística autòctona. Una producció autòctona que tal vegada no existí. Per aquesta raó pren encara més notorietat aquesta important difusió dels quartets de Pleyel.

Precisament aquesta manca d'interès per la música de cambra ens pot ajudar a entendre que gairebé la meitat dels seus quartets de corda fossin coneguts a Catalunya per mitjà de reduccions a tecla. I el mateix podem dir dels duets. Curiosament, però, cap dels seus quintets de corda no va ser objecte d'aquestes transformacions en el medi sonor, de manera que aquestes obres testimonien una pràctica de música de cambra que no podríem deduir de la producció autòctona catalana.

Altres canvis significatius són les reduccions a tecla d'algunes de les seves simfonies com també les re-instrumentacions per a dos violins d'algunes composicions per a piano i violí. En resum, i malgrat que - tal com dèiem - la seva música original per a tecla no es va conèixer a Catalunya, aproximadament la meitat de les còpies catalanes d'obres de Pleyel són per al teclat, la major part de les quals és conservada a Montserrat. De fet, gairebé la meitat de les obres de Pleyel conservades a Catalunya són a Montserrat.

L'altra tendència clara que s'observa en aquest repertori és la de fragmentar les obres en moviments separats, a vegades agrupant-los amb altres moviments isolats que provenen d'altres composicions, o fins i tot que són d'altres compositors. Entre aquests hi abunden especialment els moviments en forma sonata, els minuets i els rondós. Sembla que aquest fet està relacionat amb els gèneres $i$ instrumentacions, $i$, fins a cert punt, amb els arxius, ja que aquesta fragmentació és més freqüent en uns arxius que en altres. Això és especialment comú entre els duets i els trios, i afecta en menor mesura a les simfonies i quartets. Per altra banda, aquesta és una manera de tractar les composicions - desmuntar, reorganitzar i, en definitiva, fer «a mida»- que en major

4. Carrato Mena, M. A., Catálogo de Impresos Musicales del Siglo XVIII en la Biblioteca Nacional. Madrid, 1989. 5. VILAR, J. M., Les simfonies de Carles Baguer: fonts, context $i$ estil. Tesi Doctoral. U.A.B. Octubre, 1994. 
o menor grau afecta gairebé tota la música de tecla del classicisme, i en menor mesura la simfònica, de manera especial en el territori català.

A manca d'estudis més aprofundits, de moment suposem que la societat catalana, el gust musical a Catalunya, té una predil-leció notable per les composicions instrumentals d'un sol moviment, les raons de la qual seria difícil de resseguir. Aquesta tendència és especialment forta en la música per a tecla. Segurament que un dels compositors més ben coneguts del classicisme català madur és - fins al moment - Carles Baguer i a ell ens referim com a exemple il.lustratiu del que diem: una part considerable de les seves simfonies conservades en versió orquestral són en un sol moviment; gairebé totes llurs transcripcions per a tecla són en un sol moviment, i absolutament totes aquelles que només ens han arribat en versió per a tecla consten d'un únic temps.

Des d'aquest punt de vista, doncs, allò que els usuaris de la música d'Ignaz Pleyel fan amb ella és transformar-la i mediatitzar-la per adaptar-la als seus usos i costums, fer-la més funcional. Resta per veure fins a quin punt aquesta mediatització fou feta a Catalunya o ja era incorporada en les partitures que arribaven de l'estranger.

Pel que fa als arxius hem de dir que a Cervera no hi ha moviments aïllats, mentre que a l'altre extrem trobem que a La Seu d'Urgell i a Vic, gairebé no hi ha obres de més d'un moviment que siguin completes. A Vic (es tracta d'un llibre d'orgue de principis del segle XIX) tots els moviments d'obres que en tenien 3 o 4 foren considerats composicions separades, tot i que molts d'ells estan juxtaposats i conserven els seus títols originals.

La gran majoria de les còpies impreses arribaven a Catalunya a través de França, tal com ho testimonien les mateixes portades o bé els segells estampats a sobre. Sens dubte que aquest és un fet inherent a l'obra de Pleyel i a la difusió de què va ser objecte arreu. En alguns casos hem pogut establir que algunes de les còpies manuscrites estan basades en aquests impresos. La majoria d'aquelles són contemporànies, i les úniques excepcions corresponen als arxius de Montserrat i Olot en els quals es troben algunes còpies posteriors.

Tots els arxius són eclesiàstics excepte el fons de la Biblioteca de Catalunya. Però els documents que contenen les obres de Pleyel també tenen un origen que, pràcticament amb tota seguretat, és eclesiàstic. Ja en ocasions anteriors s'ha tractat - amb visions no sempre del tot coincidents - el tema de les funcions que devien tenir en el classicisme català les transcripcions per a tecla $a^{6}$. Sembla que entre aquestes hi devia haver la pedagògica i les interpretacions - un cop fragmentades $i$, arribat el cas, reagrupades-com a «versos» durant les cerimònies litúrgiques. La música orquestral i possiblement també els quartets i quintets devien trobar el seu destí a les Siestas i en altres casos també com a versos orquestrals.

És evident que hi havia, també, una presència que devia ser força continuada de l'obra de Pleyel en els àmbits civils de Barcelona, especialment pel fet que els músics que treballaven en els àmbits religiós, teatral i civil-privat o semi-públic eren - tot i tenint en compte que es

6. BAGUER, C., Tres sinfonias para tecla. Revisión y prólogo Maria A. Ester-Sala. Barcelona: CSIC, 1984. VILAR, J. M., Op. cit. p. 127-128. 
tractava d'una ciutat considerablement gran - fins a cert punt els mateixos. Tant d'aquesta unicitat de repertoris per mitjà dels diferents àmbits com del fet concret de la presència d'obres de Pleyel en aquests cercles en deixa bona constància el Baró de Maldà en el seu Calaix de sastre?.

Resumint, doncs, del contingut dels manuscrits i impresos estudiats i de la seva interpretació, en podem extreure les següents conclusions:

1. Ignaz Pleyel va ser un compositor àmpliament conegut a la Catalunya de finals del segle XVIII i primeres dècades del segle XIX.

2. Aquest coneixement devia revertir en una influència sobre els compositors catalans.

3. D'altra banda, aquest coneixement de l'obra de Pleyel va estar molt mediatitzat i fins i tot desfigurat per aquests canvis en la instrumentació, que tendeixen invariablement a la reducció - gairebé sempre a tecla-, i a l'esmicolament i la fragmentació que afecten moltes de les obres trobades (un $40 \%$ de les còpies catalanes contenen obres incompletes).

Aquest estat de coses sembla indicar que Catalunya no va aconseguir assimilar totalment aquest repertori centreuropeu, si més no en el seu format original, al temps que mostrava un interès per adaptar-lo a unes estructures musicals, litúrgiques, socials i probablement també mentals que eren molt diferents d'aquelles que constituïren el context natural original d'aquell repertori. Pleyel es mou en els grans centres del clasicisme musical i s'integra plenament en el mainstream d'aquest estil. Per la seva banda Barcelona i la resta de Catalunya viuen el classicisme de manera perifèrica. No és estrany, doncs, que una assimilació àmplia -moderadament àmplia almenys - de la seva obra exigís una transformació i adequació tan profunda com la que es va donar i que tal vegada ens fa qüestionar fins a quin punt el concepte de timbre i el concepte d'obra eren igual o almenys semblants als centres de decisió i a la perifèria.

4. Un estudi comparat de l'obra de Pleyel conservada a Catalunya posaria de manifest fins a quin punt aquestes composicions s'erigiren en models per als compositors del país i en companyia de quins altres repertoris i autors s'interpretaren aquestes obres.

Al final presentem una llista d'obres - amb els corresponents incipits musicals - que probablement són espúries. Totes són atribuïdes a Pleyel segons els manuscrits, però cap d'elles no apareix al catàleg Benton. De la llista inicial s' han retirat alguns incipits dels quals s' ha pogut establir que l'autor veritable era Haydn. Pel que fa a la resta, fins al moment no ens ha estat possible d'establir si aquestes falses atribucions s'originen a Catalunya, la qual cosa podria induir a pensar que els seus autors reals fossin catalans o bé si aquests errors ja s'havien comès abans que les fonts arribessin al país.

7. Vegeu, a tall d'exemples els següents:

Amat I DE Cortada, R. d', Calaix de sastre. Barcelona, Curial, 1994. Vol. 6, p. 195 (corresponent a 23 de març de 1803). Se'ns parla de la «música en una simfonia concertant, penso que de Pleyel -en los obligats de viola de Jeroniet, $i$ en violí de mossèn Josep Prats, i això també en los demés instruments- per lo summament harmoniosa». És clar que «viola» en paraules del cronista és violoncel $i$, per tant, si realment es tractava d'una obra de Pleyel, hauria estat una transcripció.

BARÓ DE MALDÀ, Exili de Barcelona i viatge a Vic (1808). Abadia de Montserrat, 1991, p. 119. En aquells moments no té «humor ab bona consonància per tocar-hi minuets, adàgios y alegros de algun concert de Hayden, o de Pleyel». Cal notar, a més, que queda ben reflectit aquell costum de fragmentar les obres en els seus respectius moviments. 


\section{Llista d'obres localitzades}

\begin{tabular}{|c|c|c|c|c|c|c|c|c|}
\hline N. ${ }^{\circ}$ & Pàg. & Títol original & Arxiu & Signatura & Títol & Instrumentació & Còpia & Notes \\
\hline 103 & 1 & Concert violí & MO & 1651 & Concerto & Orquestra & Ms. & \\
\hline \multirow[t]{2}{*}{111} & 16 & Simfonia concertante & SUe & - & Andante & Tecla & Ms. & només 2on. mov. \\
\hline & & & MO & 1650 & Noveno (sic) & Orquestra & Ms. - & Títol: «Noveno o Divertissement» \\
\hline 121 & 27 & Simfonia en do & MO & 1654 & Simfonia & Orquestra & Ms. & \\
\hline 123 & 29 & Simfonia en Fa & CE & - & Simfonia & Orquestra & Ms. & \\
\hline \multirow[t]{5}{*}{124} & 30 & Simfonia en $\operatorname{Re}$ & $\mathrm{CE}$ & - & Simfonia & Orquestra & Ms. & \\
\hline & & & MO & $547 / 18-19$ & Rondó & Tecla & Ms. & només 4rt mov. \\
\hline & & & MO & 1666 & Simfonia & Orquestra & Ms. & \\
\hline & & & MO & $2197 / 36$ & Adagio & Tecla & Ms. & només 2on mov. \\
\hline & & & MO & $1879 / 9$ & Minuet & $v 1, v 1$ & Ms. & \\
\hline \multirow[t]{3}{*}{125} & 31 & Simfonia en Si b. & $\mathrm{Bp}$ & 1599 & Simfonia & Orquestra & Ms. & \\
\hline & & & $\mathrm{Bc}$ & 845 & Minuet & Orquestra & Ms. & Només 3er mov. \\
\hline & & & $G$ & - & Simfonia & Orquestra & Ms. & \\
\hline 126 & 32 & Simfonia en $\mathrm{Re}$ & MO & 1656 & Simfonia & Orquestra & Ms. & \\
\hline \multirow[t]{5}{*}{127} & 33 & Simfonia en Si b. & $\mathrm{Bp}$ & 1589 & Simfonia & Orquestra & Ms. & \\
\hline & & & VPasm & nprov. 84 & Simfonia & Orquestra & Ms. & \\
\hline & & & MO & $2398 / 17-26$ & Simfonia & Tecla & Ms. & \\
\hline & & & $\mathrm{Bc}$ & M845 & Minuet & Orquestra & Ms. & només 3er mov. \\
\hline & & & MO & 1662 & Simfonia & Orquestra & Ms. & \\
\hline \multirow[t]{2}{*}{128} & 34 & Simfonia en Do & $\mathrm{MO}$ & $1232 / 22$ & Minuet & Orquestra & Ms. & només 3er mov. \\
\hline & & & MO & 1664 & Simfonia & Orquestra & Ms. & \\
\hline 130 & 36 & Simfonia en Sol & MO & 1655 & Simfonia & Orquestra & Ms. & \\
\hline 132 & 39 & Simfonia en Si b. & VPasm & nprov. 132 & Simfonia & Orquestra & Ms. & només ler mov. seguit de $N^{\circ} 147$ \\
\hline \multirow[t]{7}{*}{133} & 40 & Simfonia en $\mathrm{Re}$ & $\mathrm{Bc}$ & M845 & Minuet & Orquestra & Ms. & només 3er mov. \\
\hline & & & $\mathrm{MO}$ & $1232 / 20$ & Minuet & Tecla & Ms. & només 3er mov. \\
\hline & & & MO & RRR 39/1 & Sonata & Tecla & Ms. & $20 n$ mov. com a ler de Sonata 5 \\
\hline & & & MO & $2574 / 21-30$ & Simfonia & Tecla & Ms. & ler, 2on i 3er movs. \\
\hline & & & MO & $1630 / 12-15$ & Simfonia & Tecla & Ms. & només ler mov. \\
\hline & & & MO & $2435 / 15-17$ & Simfonia & Tecla & Ms. & només ler mov. \\
\hline & & & MO & 1669 & Simfonia & Orquestra & Ms. & \\
\hline 134 & 42 & Simfonia en Mi b. & Mo & 1657 & Simfonia & Orquestra & Ms. & \\
\hline \multirow[t]{4}{*}{135} & 46 & Simfonia en Si b. & Bp & M1586 & Sonatina & fortepiano & Ms. & només 4rt mov. \\
\hline & & & MO & $1633 / 15-17$ & Rondó & Tecla & Ms. & només $4 \mathrm{rt}$ mov. \\
\hline & & & MO & RRR 39/1 & Rondó & Tecla & Ms. & $4 \mathrm{rt}$ mov. com a $20 n$ de Sonata 3 \\
\hline & & & MO & 1658 & & Orquestra & Ms. & Font probable: (1616) \\
\hline \multirow[t]{5}{*}{136} & 48 & Simfonia en Fa & VPasm & nprov. 85 & Simfonia & Orquestra & Ms. & Font probable: (1616) \\
\hline & & & $\mathrm{Bc}$ & M 1578 & Simfonia & Orquestra & Ms. & Font probable: (1616) \\
\hline & & & $\mathrm{MO}$ & $1630 / 1-4$ & Sonata & Tecla & Ms. & només ler inov. \\
\hline & & & MO & $2435 / 3-7$ & Sonata & Tecla & Ms. & només ler mov. \\
\hline & & & MO & 1659 & Simfonia & Orquestra & Ms. & \\
\hline \multirow[t]{4}{*}{137} & 50 & Simfonia en La & MO & $568 / 17$ & Minuet & Orgue & Ms. & Especificat: «para corneta» \\
\hline & & & MO & $1292 \mathrm{~b} / 13-14$ & 4Rondó & Tecla & Ms. & només 4rt mov. \\
\hline & & & MO & RRR 39/1 & Sonata & Tecla & Ms. & $3 \mathrm{er}, 2 \mathrm{on} \mathrm{i} 4 \mathrm{rt}$ mov. \\
\hline & & & MO & 1660 & Simfonia & Orquestra & Ms. & \\
\hline \multirow[t]{3}{*}{138} & 54 & Simfonia en fa & $\mathrm{CE}$ & - & Simfonia & Orquestra & Ms. & \\
\hline & & & $\mathrm{MO}$ & RRR 39/1 & Sonata & Tecla & Ms. & 3rer i 4rt mov. com a 2 on i 3er de Sonata 5 \\
\hline & & & MO & 1652 & Simfonia & Orquestra & Ms. & \\
\hline 139 & 55 & Simfonia en Mi b. & MO & $566 / 34-39$ & Simfonia & Tecla & Ms. & només ler mov. \\
\hline \multirow[t]{2}{*}{140} & 56 & Simfonia en $\mathrm{Fa}$ & OLp & - - & Minuet & Orquestra & Ms. & només 3er mov. \\
\hline & & & $\mathrm{MO}$ & 1649 & Andante & Orquestra & Ms. & Títol: Andante para la Comunión; només 3er mov. \\
\hline $140 \mathrm{~A}$ & 56 & Simfonia en $\mathrm{Fa}$ & VIG & c. 7 & Andante & Tecla & Ms. & Seguit d'un minuet de Mateu Ferrer \\
\hline
\end{tabular}




\begin{tabular}{|c|c|c|c|c|c|c|c|c|}
\hline N..$^{\circ}$ & Pàg. & Títol original & Arxiu & Signatura & Títol & Instrumentació & Còpia & Notes \\
\hline \multirow[t]{2}{*}{142} & 58 & Simfonia en do & $\mathrm{CE}$ & - & Simfonia & Orquestra & Ms. & \\
\hline & & & MO & 1661 & Simfonia & Orquestra & Ms. & \\
\hline \multirow[t]{2}{*}{143} & 59 & Simfonia en Do & $\mathrm{CE}$ & - & Simfonia & Orquestra & Ms. & \\
\hline & & & MO & 1668 & Simfonia & Orquestra & Ms. & \\
\hline 144 & 60 & Simfonia en Mi b. & $\mathrm{CE}$ & - & Simfonia & Orquestra & Ms. & \\
\hline \multirow[t]{5}{*}{145} & 60 & Simfonia en Re & $\mathrm{CE}$ & - & Simfonia & Orquestra & Ms. & \\
\hline & & & MO & $1630 / 8-12$ & Sonata & Tecla & Ms. & només ler mov. \\
\hline & & & MO & $2435 / 12-15$ & 5 Sonata & Tecla & Ms. & només ler mov. \\
\hline & & & VPasm & nprov. 86 & Simfonia & Orquestra & Ms. & Font probable: (1773) \\
\hline & & & OLp & - & Minuet & Orquestra & Ms. & només 3er mov. \\
\hline \multirow[t]{2}{*}{146} & 61 & Simfonia en Sol & $\mathrm{CE}$ & - & Simfonia & Orquestra & Ms. & \\
\hline & & & MO & 1667 & Simfonia & Orquestra & Ms. & \\
\hline \multirow[t]{6}{*}{147} & 62 & Simfonia en re & $\mathrm{CE}$ & - & Simfonia & Orquestra & Ms. & \\
\hline & & & $\mathrm{Bp}$ & 1591 & Simfonia & Orquestra & Ms. & \\
\hline & & & Bp & 1587 & Simfonia & Orquestra & Ms. & \\
\hline & & & VPasm & nprov. 87 & Simfonia & Orquestra & Ms. & ler, 2on i 3er movs. \\
\hline & & & VPasm & nprov. 132 & Simfonia & Orquestra & Ms. & Només Minuet després del ler mov. de $\mathrm{N}^{\circ} 132$ \\
\hline & & & $\mathrm{Bc}$ & M 845 & Minuet & Orquestra & Ms. & \\
\hline 150 & 64 & Simfonia en Si b. & MAs & 749 & Simfonia & Orquestra & Ms. & Font probable: $(1618)$ o $(1818)$ \\
\hline 156 & 66 & Simfonia en Sol & $\mathrm{Bc}$ & M 1579 & Simfonia & Orquestra & Ms. & \\
\hline 209 & 73 & Minuet i Trio & MO & RRR 39/1 & Minuet & Tecla & Ms. & Títol: Minueto $1^{\circ}$ \\
\hline 210 & 73 & Minuet i Trio & MO & RRR 39/1 & Minuet & Tecla & Ms. & Títol: Minueto $2^{\circ}$ \\
\hline 211 & 73 & Minuet i Trio & MO & RRR 39/1 & Minuet & Tecla & Ms. & Títol: Minueto $3^{\circ}$ \\
\hline 212 & 73 & Minuet i Trio & MO & RRR 39/1 & Minuet & Tecla & Ms. & Títol: Minueto $4^{\circ}$ \\
\hline 213 & 73 & Minuet i Trio & MO & RRR 39/1 & Minuet & Tecla & Ms. & Títol: Minueto $5^{\circ}$ \\
\hline 214 & 73 & Minuet i Trio & MO & RRR 39/1 & Minuet & Tecla & Ms. & Títol: Minueto $6^{\circ}$ \\
\hline \multirow[t]{3}{*}{271} & 83 & Quintet en Mi b. & $\mathrm{CE}$ & - & Quintet & Quintet & Im. & Font: (2505) \\
\hline & & & MO & 1646 & Quintet & Quintet & Ms. & \\
\hline & & & MO & 1648 & Quintet & Quintet & Ms. & \\
\hline \multirow[t]{3}{*}{272} & 83 & Quintet en sol & $\mathrm{CE}$ & - & Quintet & Quintet & Im. & Font: (2505) \\
\hline & & & MO & 1646 & Quintet & Quintet & Ms. & \\
\hline & & & MO & 1648 & Quintet & Quintet & Ms. & \\
\hline \multirow[t]{3}{*}{273} & 83 & Quintet en Do & $\mathrm{CE}$ & - & Quintet & Quintet & Im. & Font: (2505) \\
\hline & & & MO & 1646 & Quintet & Quintet Ms. & & \\
\hline & & & MO & 1648 & Quintet & Quintet & Ms. & \\
\hline \multirow[t]{4}{*}{274} & 87 & Quintet en $\mathrm{Re}$ & $\mathrm{CE}$ & - - & Quintet & Quintet & Im. & Font: (2544) \\
\hline & & & MO & $1629 / 8-10$ & Adagio & Tecla & Ms. & només 2 on mov. \\
\hline & & & MO & $1631 / 5-16$ & Sonata & Tecla & Ms. & \\
\hline & & & MO & 1647 & Quintet & Quintet & Ms. & \\
\hline \multirow[t]{2}{*}{275} & 87 & Quintet en Si b & $\mathrm{CE}$ & - & Quintet & Quintet & $\mathrm{Im}$. & Font: (2544) \\
\hline & & & MO & 1647 & Quintet & Quintet & Ms. & \\
\hline \multirow[t]{2}{*}{276} & 87 & Quintet en la & $\mathrm{CE}$ & - & Quintet & Quintet & Im. & Font: (2544) \\
\hline & & & MO & 1647 & Quintet & Quintet & Ms. & \\
\hline 278 & 90 & Quintet en Si b. & $\mathrm{CE}$ & - & Quintet & Quintet & Im. & Font: (2568) \\
\hline 279 & 90 & Quintet en Sol & $\mathrm{CE}$ & $\ldots$ & Quintet & Quintet & Im. & Font: (2568) \\
\hline \multirow[t]{2}{*}{280} & 92 & Quintet en Sol & MO & $1290 / 8$ & Andante & Tecla & Ms. & només 2 on mov. \\
\hline & & & MO & RRR 39/1 & Andante & Tecla & Ms. & 2on mov. com a ler de Sonata 1 \\
\hline \multirow[t]{4}{*}{301} & 99 & Quartet in Do & $\mathrm{CE}$ & - & Quartet & Quartet & Ms. & Font probable: (3009) \\
\hline & & & TAG & - & Quartet & Quartet & Ms. & \\
\hline & & & VI & $71-73$ & Quartet & Tecla & Ms. & \\
\hline & & & SJ & - & Quartet & Quartet & Ms. & \\
\hline \multirow[t]{3}{*}{302} & 99 & Quartet en Mi b. & $\mathrm{CE}$ & - & Quartet & Quartet & Ms. & Font probable: (3009) \\
\hline & & & MO & $488 / 25-26$ & Vid. Notes & Tecla & Ms. & Títol: Minuet, Adagio, Rondó \\
\hline & & & MO & $1292 b / 15-1$ & 16Minuet & Tecla & Ms. & \\
\hline
\end{tabular}




\begin{tabular}{|c|c|c|c|c|c|c|c|c|}
\hline & & & MO & $1929 a / 51$ & Minuet & Tecla & Ms. & \\
\hline & & & MO & $1382 / 60$ & Minuet & Tecla & Ms. & L'autor és Haydn, segons el Ms. \\
\hline & & & MO & $488 / 33-37$ & Sonata & Tecla & Ms. & només ler mov. \\
\hline & & & TAG & - & Quartet & Quartet & Ms. & \\
\hline & & & VI & $74,76,75$ & Quartet & Tecla & Ms. & ler, 2on i 3er mov. \\
\hline & & & SJ & - & Quartet & Quartet & Ms. & \\
\hline 303 & 99 & Quartet en La & $\mathrm{CE}$ & - & Quartet & Quartet & Ms. & Font probable: (3009) \\
\hline & & & TAG & - - & Quartet & Quartet & Ms. & \\
\hline & & & VI & 77,78 & Quartet & Tecla & Ms. & ler, 2on i 3er mov. \\
\hline & & & SJ & - & Quartet & Quartet & Ms. & \\
\hline 304 & 99 & Quartet en Si b. & $\mathrm{CE}$ & - & Quartet & Quartet & Ms. & Font probable: (3009) \\
\hline & & & TAG & - - & Quartet & Quartet & Ms. & \\
\hline & & & VI & $79-81$ & Quartet & Tecla & Ms. & \\
\hline & & & SJ & - & Quartet & Quartet & Ms. & \\
\hline 305 & 99 & Quartet en Sol & $\mathrm{CE}$ & - & Quartet & Quartet & Ms. & Font probable: (3009) \\
\hline & & & TAG & - & Quartet & Quartet & Ms. & \\
\hline & & & VI & $82-84$ & Quartet & Tecla & Ms. & \\
\hline & & & SJ & - - & Quartet & Quartet & Ms. & \\
\hline 306 & 99 & Quartet en Re & $\mathrm{CE}$ & - & Quartet & Quartet & Ms. & Font probable: (3009) \\
\hline & & & TAG & - & Quartet & Quartet & Ms. & \\
\hline & & & VI & $85-87$ & Quartet & Tecla & Ms. & \\
\hline 307 & 106 & Quartet en La & $\mathrm{CE}$ & - & Quartet & Quartet & Ms. & \\
\hline & & & VI & $88-90$ & Quartet & Tecla & Ms. & \\
\hline & & & $\mathrm{Bc}$ & M 845 & Minuet & Orquestra & Ms. & \\
\hline & & & MO & 1642 & Quartet & Quartet & Ms. & \\
\hline 308 & 106 & Quartet en Do & $\mathrm{CE}$ & - & Quartet & Quartet & Ms. & \\
\hline & & & VI & $91-93$ & Quartet & Tecla & Ms. & \\
\hline & & & MO & 1642 & Quartet & Quartet & Ms. & \\
\hline 309 & 106 & Quartet en sol & $\mathrm{CE}$ & - & Quartet & Quartet & Ms. & \\
\hline & & & VI & $94-96$ & Quartet & Tecla & Ms. & \\
\hline & & & MO & 1642 & Quartet & Quartet & Ms. & \\
\hline 310 & 106 & Quartet en Mi b. & $\mathrm{CE}$ & - & Quartet & Quartet & Ms. & \\
\hline & & & VI & $97-99$ & Quartet & Tecla & Ms. & \\
\hline & & & MO & 1642 & Quartet & Quartet & Ms. & \\
\hline 311 & 106 & Quartet en Si b. & $\mathrm{CE}$ & - - & Quartet & Quartet & Ms. & \\
\hline & & & VI & $100-102$ & Quartet & Tecla & Ms. & \\
\hline & & & MO & 1642 & Quartet & Quartet & Ms. & \\
\hline 312 & 106 & Quartet en $\mathrm{Re}$ & $\mathrm{CE}$ & - - & Quartet & Quartet & Ms. & \\
\hline & & & VI & $103-104$ & Quartet & Tecla & Ms. & \\
\hline & & & MO & 1642 & Quartet & Quartet & Ms. & \\
\hline 313 & 111 & Quartet en Si b. & VI & $119-122$ & Quartet & Tecla & Ms. & \\
\hline 314 & 111 & Quartet en La & VI & $123-124$ & Quartet & Tecla & Ms. & Ier i $20 n$ mov. \\
\hline 319 & 114 & Quartet en Do & $\mathrm{CE}$ & - & Quartet & Quartet & Im. & Font: $(3154)$ \\
\hline & & & VI & $105-106$ & Quartet & Tecla & Ms. & \\
\hline 320 & 114 & Quartet en Sol & $\mathrm{CE}$ & - & Quartet & Quartet & $\mathrm{Im}$. & Font: $(3154)$ \\
\hline & & & VI & $107-109$ & Quartet & Tecla & Ms. & \\
\hline 321 & 114 & Quartet en Fa & $\mathrm{CE}$ & - & Quartet & Quartet & $\operatorname{lm}$. & Font: $(3154)$ \\
\hline & & & VI & $22+110$ & Quartet & Tecla & Ms. & \\
\hline 322 & 114 & Quartet en La & $\mathrm{CE}$ & - & Quartet & Quartet & Im. & Font: $(3154)$ \\
\hline & & & VI & $111-112$ & Quartet & Tecla & Ms. & ler i 3er mov. \\
\hline & & & Bac & $4 / 8$ & Andante & Tecla & Ms. & només ler mov. \\
\hline & & & $\mathrm{Bc}$ & M 845 & Minuet & Orquestra & Ms. & només 3er mov. \\
\hline & & & SJ & - & Quartet & Quartet & Ms. & \\
\hline 323 & 114 & Quartet en Si b. & $\mathrm{CE}$ & - & Quartet & Quartet & Im. & Font: (3154) \\
\hline & & & VI & $113-115$ & Quartet & Tecla & Ms. & \\
\hline 324 & 114 & Quartet en $\mathrm{Re}$ & $\mathrm{CE}$ & - & Quartet & Quartet & $\operatorname{lm}$. & Font: $(3154)$ \\
\hline
\end{tabular}




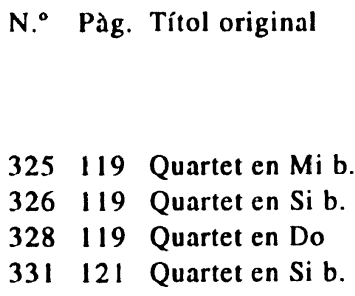

332121 Quartet en Sol

333122 Quartet en re 334122 Quartet en Do

335122 Quartet en L

336122 Quartet en Mi b.

$337 \quad 122$ Quartet en Re

338122 Quartet en Fa

339122 Quartet en Si b.

340122 Quartet en Sol

$341 \quad 122$ Quartet en do

342123 Quartet en Re

343136 Quartet en Fa

346137 Quartet en Do

347137 Quartet en Fa
Arxiu Signatura Títol

Bc M 845 Minuet

MO 1292b/17-183Quartet

MO 574/58 Allegro

Bp $1586 \quad$ Sonatina

CE - $\quad$ Quartet

G LXV, 17 Sonata

MO 163lb/28-33Sonata

MO 1631/24-32 Sonata

MO RRR 39/1 Adagio

$\mathrm{CE}$

B



CE

VI

MO 1232/17 Allegro

MO 479/8-9 Allegro

MO 1107/6 Allegro

MO 2509/28-29 Allegro

Bp 1525 Rondo

SUe - Quartet

Bc M 932/7 Rondó

MO RRR 39/1 Sonata

MO RRR 39/1 Sonata

VI 57-58 Quartet

OLP 410

CE -

VI 50-5I

VI 61

Variacions

Quartet

Quartet

MO 2509/28-29 Allegro

VI $59-60 \quad$ Quartet

VI $43 \quad$ Romance

MO RRR 39/1 Romance

MO RRR 39/1 Rondó

SUe - $\quad$ Trio

Bp $1586 \quad$ Sonatina

MO 1635/18-22 Rondó

MO 1629/7-8 Rondó

MO 1629/11-12 Sonata

MO 1631/32-41 Sonata

MO RRR 39/1 Rondó

MO $1644 \quad$ Quartet

MO 1665/1 Rondó

Bc M 583 Quartet

Bp M 1546 Quartet

MO 1292a/50 Rondó

MO 1629/4-6 Rondó

MO 577/15-20 Sonata

MO 1382/98-99 Sonata

MO 1382/102-3 Sonata
Instrumentació Còpia Notes

Tecla Ms.

Orquestra Ms. només 3er mov.

Tecla Ms. només ler mov.

Tecla Ms. només ler mov.

piano Ms.

Quartet Ms

piano

Tecla

Tecla

Tecla

Tecla

Quartet

Tecla

Tecla

piano

Tecla

Quartet

Quartet

Tecla

Tecla

Tecla

Tecla

Tecla

Tecla

Quartet

Tecla

Tecla

Tecla

Tecla

Tecla

Quartet

Tecla

Tecla

Tecla

Tecla

Tecla

Tecla

Tecla

g, vl, vla

Tecla

Tecla

Tecla

Tecla

Tecla

Tecla

Quartet

Quartet

Quartet

Quartet

Tecla

Tecla

Tecla

Tecla

Tecla

ler mov. només

Ms.

Ms. Ms.

Ms.

Ms. només ler mov.

Ms.

Ms.

Ms.

Ms.

Ms.

Ms.

3er mov. seguit

Ms.

Ms.

només 3er mov.

Ms.

Ms.

Ms.

Ms.

Ms.

Ms.

Ms. ler i 2on mov.

Ms. només 2on mov.

Ms. ler i 3er mov.

Ms. només 20 mov.

Ms. només 3er mov.

Ms. només ler mov.

Ms.

Ms.

Ms.

Ms.

Ms.

Ms. només 3er mov.

Ms. només 3er mov.

Ms. només ler mov.

Ms. només ler mov.

Ms. només ler mov. només 2on mov. com a ler

Ms. 2on mov. com a ler de Sonata 3

Ms. ler i 3er mov. només i ler mov. de $\mathrm{N}^{\circ} 331$

Ms. 3er mov. com a 20 n

2on i 3er mov. com a ler i 20 de Sonata 4

Ms. 2on mov. after 3er mov. de $\mathrm{N}^{\circ} 334$

Ms. 2on mov. com a ler de Sonata 4

Ms. 3er mov. com a ler de Sonata 5

Ms. només 3er mov. com a 20 n

Ms. 3er mov. com a 2 on de Sonata 2 
N. ${ }^{\circ}$ Pàg. Títol original

\begin{tabular}{|c|c|c|c|c|c|}
\hline & & & MO & $1382 / 106-9$ & Sonata \\
\hline & & & MO & $1629 / 37-40$ & Sonata \\
\hline & & & MO & $1631 / 42-46$ & Sonata \\
\hline & & & $\mathrm{Bc}$ & M 583 & Quartet \\
\hline & & & MO & 1644 & Quartet \\
\hline 348 & 138 & Quartet en Si b. & $\mathrm{Bp}$ & 1546 & Quartet \\
\hline & & & $\mathrm{Bc}$ & M 583 & Quartet \\
\hline & & & MO & 1644 & Quartet \\
\hline 349 & 138 & Quartet en Sol & SUe & - & Trio \\
\hline & & & $\mathrm{Bc}$ & M 583 & Quartet \\
\hline & & & MO & $1637 / 22$ & Quartet \\
\hline & & & MO & 1644 & Quartet \\
\hline 350 & 138 & Quartet en Si b. & MO & $1291 / 5-6$ & Rondó \\
\hline & & & $\mathrm{Bc}$ & M 583 & Quartet \\
\hline & & & MO & RRR 39/1 & Andante \\
\hline & & & MO & 1644 & Quartet \\
\hline 351 & 138 & Quartet en La & $\mathrm{Bc}$ & M 845 & Minuet \\
\hline & & & $\mathrm{Bc}$ & M 583 & Quartet \\
\hline & & & MO & 1644 & Quartet \\
\hline 352 & 145 & Quartet en Mi b. & MO & 1643 & Quartet \\
\hline 353 & 147 & Quartet en Do & $\mathrm{CE}$ & - & Quartet \\
\hline & & & MO & $1250 / 44-47$ & Rondó \\
\hline & & & MO & 1640 & Quartet \\
\hline 354 & 147 & Quartet en Si b. & $\mathrm{CE}$ & - & Quartet \\
\hline & & & MO & $1250 / 53-57$ & All./Min. \\
\hline & & & MO & $574 / 65-66$ & Minuet \\
\hline & & & MO & 1640 & Quartet \\
\hline 355 & 147 & Quartet en mi & $\mathrm{CE}$ & - - & Quartet \\
\hline & & & MO & $1250 / 50-53$ & Allegro \\
\hline & & & MO & $1250 / 47-49$ & Allegro \\
\hline & & & MO & 1640 & Quartet \\
\hline 356 & 147 & Quartet en Sol & MO & 1641 & Quartet \\
\hline 357 & 147 & Quartet en La & MO & 1641 & Quartet \\
\hline 358 & 147 & Quartet en fa & MO & $1382 / 63$ & Minuet \\
\hline & & & MO & 1641 & Quartet \\
\hline 387 & 168 & Quartet en $\mathrm{Re}$ & MO & $1632 / 8-10$ & Polonesa \\
\hline 391 & 168 & Quartet en Sol & MO & $1632 / 3-7$ & Rondó \\
\hline 395 & 169 & Quartet en Mi b. & Bo & 1586 & Sonatina \\
\hline & & & SUe & - & Sonatina \\
\hline & & & MO & $577 / 12-15$ & Andante \\
\hline & & & MO & RRR 39/1 & Andante \\
\hline & & & MO & $1109 / 17-18$ & Andante \\
\hline & & & MO & $1635 / 23-26$ & Andante \\
\hline & & & MO & $1636 / 2-5$ & Andante \\
\hline 401 & 175 & Trio en Mi b. & $\mathrm{CE}$ & - - & Trio \\
\hline & & & MO & $572 / 9-16$ & Trio \\
\hline 402 & 175 & Trio en $\mathrm{Re}$ & $\mathrm{CE}$ & - & Trio \\
\hline & & & MO & $572 / 19-23$ & Trio \\
\hline 403 & 175 & Trio en $\mathrm{Fa}$ & $\mathrm{CE}$ & - & Trio \\
\hline & & & MO & $572 / 24$ & Trio \\
\hline 404 & 178 & Trio en Do & MO & RRR 39/1 & Rondó \\
\hline & & & MO & 1639 & Trio \\
\hline 40 & 178 & Trio en Mi b. & MO & 1639 & Trio \\
\hline & & & & RRR 3 & \\
\hline
\end{tabular}

\section{Instrumentació Còpia Notes}

Tecla Ms. només ler mov.

Tecla Ms. només ler mov.

Tecla Ms. només ler mov.

Quartet Ms.

Quartet Ms.

Quartet Ms.

Quartet Ms.

Quartet Ms.

g, vl, vla Ms.

Quartet Ms.

Quartet Ms.

Tecla Ms. només 2on mov.

Quartet

Tecla

Quartet

Tecla

Quartet

Quartet

Quartet

Quartet

Tecla

Quartet

Quartet

Tecla

Tecla

Quartet

Quartet

Tecla

Tecla

Quartet

Quartet

Quartet

$\mathrm{fl}, \mathrm{fl}$

Quartet

Tecla

Tecla

piano

piano

Tecla

Tecla

Tecla

Tecla

Tecla

Trio

Trio

Trio

Trio

Trio

Trio

Tecla

Trio

Trio

Tecla
Ms.

Ms. 2on mov. com a ler de Sonata 6

Ms.

Ms.

Ms.

Ms.

Ms.

Ms.

Ms. només 3er mov.

Ms.

Ms.

Ms. ler i només 2on mov.

Ms. només 2on mov.

Ms.

Ms.

Ms. nonés ler mov.

Ms. només 3er mov.

Ms.

Ms.

Ms.

Ms.

Ms.

Ms.

Ms.

només 2on mov. com a ler

Ms. només 2on mov. com a ler

Ms. només 20 mov.

Ms. 2on mov. com a ler de Sonata 2

Ms. només 2on mov.

Ms. només 2on mov.

Ms. només 20 mov.

Im. Font: (4014)

Ms.

Im. Font: (4014)

Ms. només ler mov.

Im. Font: (4014)

Ms. només ler mov.

Ms. 3er mov. com a 3er de Sonata 4

Ms.

Ms.

Ms. 2on mov. com a 20 de Sonata 2 


\section{N. Pàg. Títol original}

$407 \quad 178$ Trio en Sol

408178 Trio en Si b.

$409 \quad 188$ Trio en Sol

$431 \quad 188$ Trio en Do

$432 \quad 188$ Trio en Sol

433188 Trio en Si b.

$434 \quad 188$ Trio en La

$435 \quad 188$ Trio en mi

$436 \quad 188$ Trio en $\mathrm{Re}$

$437 \quad 199$ Trio en Fa

441200 Trio en Do

470220 Trio en Do

501235 Duet en Do

502235 Duet en Re

513240 Duet en Si b.

514240 Duet en Re

515240 Duet en La

516240 Duet en Fa

517240 Duet en Do

518240 Duet en mi

519246 Duet en Do

520246 Duet en sol
Arxiu Signatura Títol

\begin{tabular}{|c|c|c|}
\hline MO & 1639 & Trio \\
\hline MO & 1639 & Trio \\
\hline $\mathrm{CE}$ & - & Trio \\
\hline MO & RRR 39/1 & Sonata \\
\hline MO & 1639 & Trio \\
\hline $\mathrm{CE}$ & & Trio \\
\hline $\mathrm{CA}$ & - & Allegro \\
\hline 10 & 1639 & Trio \\
\hline
\end{tabular}

CE — Trio

OLp - Duet

$\begin{array}{lll}\text { VI } 28 & \text { Trio }\end{array}$

MO 1635/60 Sonata

MO 1635/6-8 Rondó

CE — $\quad$ Trio

$\begin{array}{lll}\text { OLp } & - & \text { Duet } \\ \text { CE } & - & \text { Trio }\end{array}$

OLp - Duet

MO 577/6-15 Sonata

MO 1635/23-26 Variacions

MO 1636 Variacions

CE - - Trio

$\mathrm{CE}$ - Trio

OLp - Duet

MO 1635/12-13 Andante

MO 1635/13 Rondó

CE - Trio

OLp - - Duet

MO 1635/9-11 Rondo

OLp - Duet

MO 1559/12-15 Sonata

MO 1632/1-14 Sonata

MO 1291/36-37 Sonatina

MO RRR 39/1 Minuet

ESm - - Duet

ESm - - Duet

Bp 1557 Duet

ESm - - Duet

ESm - Duet

MO RRR 39/1 Adagio

MO RRR 39/1 Minuet

ESm - - Duet

ESm - D Duet

ESm - - Duet

ESm - D Duet

ESm - - Duet

ESm - D Duet

Bp 1557 Duet

Bp 1542 Duet

ESm - - Duet

$\mathrm{ESm}$ - Duet

MO RRR 39/1 Minuet

SUe - - Duet

VIG c. 8 Duet

SUe - $\quad$ Duet
Instrumentació Copia Notes

$\begin{array}{lll}\text { Trio } & \text { Ms. } & \\ \text { Trio } & \text { Ms. } & \\ \text { Trio } & \text { Ms. } & \\ \text { Tecla } & \text { Ms. } & \text { només ler mov. } \\ \text { Trio } & \text { Ms. } & \\ \text { Trio } & \text { Ms. } & \\ \text { Tecla } & \text { Ms. } & \text { només ler mov. } \\ \text { Trio } & \text { Ms. } & \\ \text { Trio } & \text { Im. } & \text { Font probable: }(4324) \text { o (4325) } \\ \text { vl, vl } & \text { Ms. } & \text { ler i 3er mov. } \\ \text { Trio } & \text { Im. } & \text { només ler mov. } \\ \text { Orgue } & \text { Ms. } & \text { ler i 3er mov. } 1799 \\ \text { Tecla } & \text { Ms. } & \text { només 3er mov. } \\ \text { Trio } & \text { Im. } & \text { Font probable: }(4324) \text { o (4325) } \\ \text { vl, vl } & \text { Ms. } & \text { ler i 3er mov. } \\ \text { Trio } & \text { Im. } & \text { Font probable: }(4324) \text { o (4325) }\end{array}$

vl, vl

Tecla

Tecla

Tecla

Trio

Trio

vl, vl

Tecla

Tecla

Trio

Tecla

Tecla

vl, vl

Tecla

Tecla

Tecla

Tecla

vl, vl

$\mathrm{vl}, \mathrm{vl}$

$\mathrm{vl}, \mathrm{vl}$

$\mathrm{vl}, \mathrm{vl}$

vl, vl

Tecla

Tecla

$\mathrm{vl}, \mathrm{vl}$

$\mathrm{vl}, \mathrm{vl}$

$\mathrm{vl}, \mathrm{vl}$

vl, vl

$\mathrm{vl}, \mathrm{vl}$

vl, vl

$\mathrm{vl}, \mathrm{vl}$

vl, vl

vl, vl

vl, vl

Tecla

vl, vl

$\mathrm{vl}, \mathrm{vl}$

vl, vl només 20 mov.

només 2on mov.

només 2on mov.

Font probable: (4324) o (4325)

Font probable: (4324) o (4325)

ler i 3er mov.

només 20 mov.

només $3 \mathrm{er}$ mov.

Font probable: (4324) o (4325)

només 2on mov.

ler i 3er mov.

només ler mov.

només ler mov.

només 20 mov.

2on mov. com a 2on de Sonata 6

Ms.

Ms.

Ms.

Ms.

Ms. ler mov. com a ler de Sonata 1

Ms. 3er mov. com a 20 de Sonata 4

Ms.

Ms.

Ms.

Ms.

Ms.

Ms.

Ms.

Ms.

Ms.

Ms.

Ms. 3er mov. com a ler de Sonata 2

Ms.

Ms. Títol: «6 duetti...» 
N. ${ }^{\circ}$ Pàg. Títol original

521246 Duet en La

522246 Duet en Si b.

523246 Duet en Sol

524246 Duet en Fa

533259 Duet en Si b

571282 Duet en Si b.

572282 Duet en Sol

574285 Duet en Do

575285 Duet en Fa

576285 Duet en Sol

577286 Duet en Si b.

578286 Duet en $\mathrm{Re}$

579286 Duet en Mi b.

582292 Duet en Si b.
Arxiu Signatura Títol

V

Duet

OLp 410

Adagio

MO 2509/21-26 Adagio

SUe - Duet

SUe - Duet

MO 1291/35 Minuet

SUe - - Duet

OLp 410

Rondo

MO 2649/35 Rondo

SUe - - Duet

SUe - - Tema

OLp 410

MO 1638/1

Adagio/Var

Variacions

MAs 1066

Rondo

MO 1361/2-4 Sonata

MO 1633/7-10 Sonata

MO 608/116 Sonata

MO 564/16-17 Sonata

MO 652/42-44 Ad./All.

MO 1629/10-11 Ad./All.

MO RRR 39/1 Sonata

MO 2711/110 Adagio

MO 1633/1-7 Sonata

MO 1631/16-26 Sonata

MO 1291/17-23 Moderato

VIG c. $7 /$ c. 8 Duet

VIG c. $7 /$ c. 8 Duet

VIG c. $7 / c .8$

OLP

c. $7 /$ c. 8

Duet

Sonata

OLp

VIG c. $7 /$ c. 8 Duet

VIG c. $7 /$ c. 8 Duet

Bac $4 / 28$

OLp

OLp

VIG c. $7 /$ c. 8 Duet

OLp Andante

Bp $1525 \quad$ Rondó
Instrumentació Còpia Notes

vI, vI

Tecla

Tecla

vI, vl

vl, vl

Tecla

vl, vl

Tecla

$\mathrm{fl}, \mathrm{fl}$

vl, vl

vl, vl

piano

Tecla

vlc, vlc

Tecla

Tecla

Tecla

Tecla

Tecla

Tecla

Tecla

Tecla

Tecla

Tecla

Tecla

vl, vl

vI, vI

vl, vl

piano, vl

piano, vl

$\mathrm{vl}, \mathrm{vl}$

vl, vl

Tecla

piano

piano

vl, vl

piano

Tecla

Ms.

Ms.

Ms.

Ms.

Ms.

Ms.

Ms.

Ms.

Ms.

Ms.

Ms.

Ms.

Ms.

Ms.
Ms. només 2on mov. com a ler

Ms. només 2on mov.

Ms. només 3er mov.

Ms. només 2on mov. després del $20 n$ mov. de $\mathrm{N}^{\circ} 520$

Ms. només 3er mov.

Ms. ler i només 3er mov.

Ms. només 3er mov.

Ms. només 2on mov.

Ms. només ler mov.

Ms. només ler mov.

Ms. només ler mov.

Ms. 2on i només 3er mov.

Ms. 2on i només 3er mov.

Ms. només 2on mov.

Ms. només ler mov.

Ms. ler i 2on mov.

Ms. només 2on mov.

Ms. només ler mov.

Ms. només 3er mov.

Ms. només 3er mov.

Ms. 2on i només 3er mov.

Ms. només ler mov.

Ms. només ler mov.

Ms. només 3er mov. 
Incipits d'obres espúries de Pleyel localitzades en arxius catalans

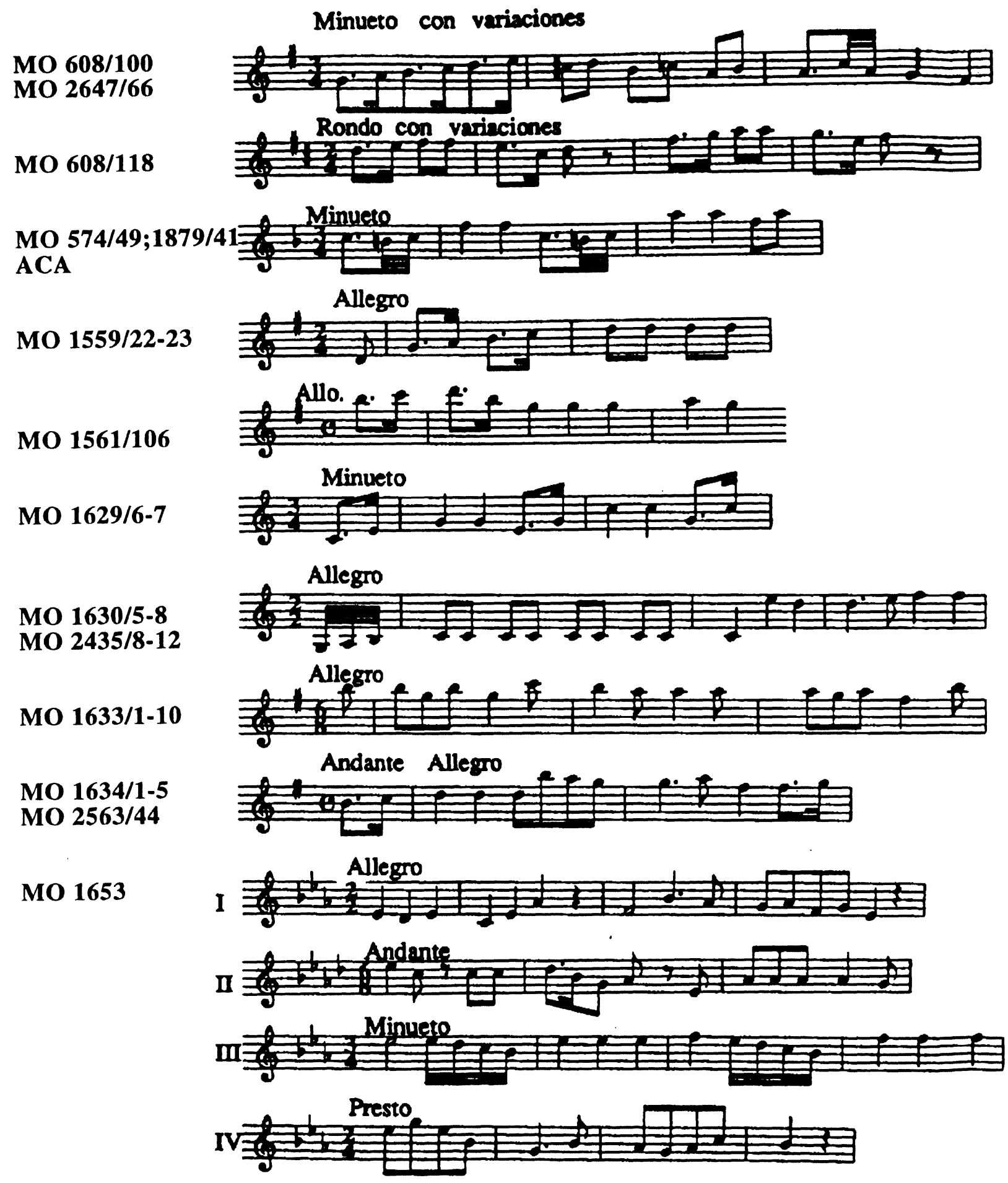

[14] 
MO RRR39/1

\section{Minueto}

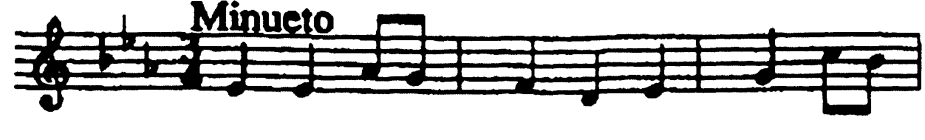

MO RRR39/1

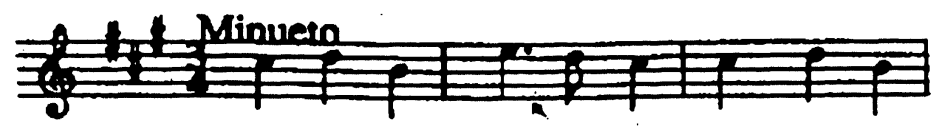

MO RRR39/1

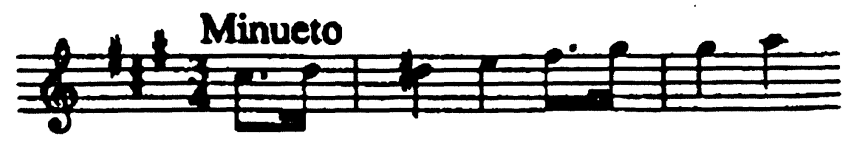

BC 932/7 p. 31

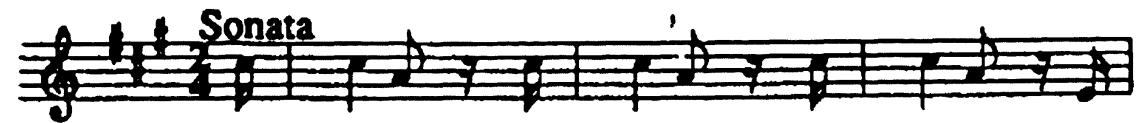

BC 932/7 p. 31

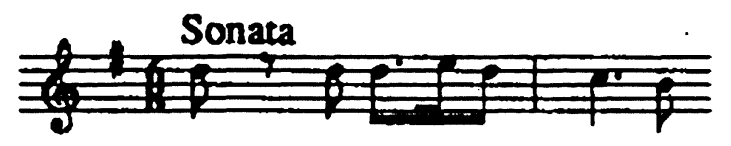

BC $932 / 7$ p. 31

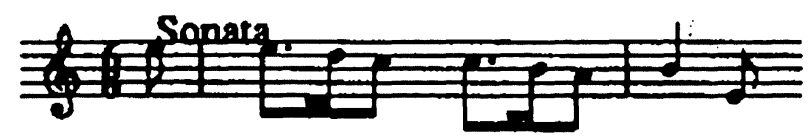

